\title{
La regulación en la calidad del trabajo de auditoría: análisis global para un contexto local (Perú)
}

\author{
Alberto Álvarez López, Lorenza Morales Alvarado \\ Facultad de Ciencias Empresariales, Universidad César Vallejo, Perú \\ Comité de Ética profesional de la Junta de Decanos de Colegios de Contadores \\ Públicos del Perú, Perú
}

\section{Resumen}

En los últimos años, los hechos ocurridos respecto de fraude y corrupción han originado que los países busquen mecanismos que salvaguarden la información financiera, en primer lugar, a través del cumplimiento de normas emitidas por organismos como IFAC, que promueve la aplicación de las Normas Internacionales de Información Financiera (NIIF), las Normas Internacionales de Auditoría (NIA) y las Normas Internacionales de Control de Calidad (NICC). Bajo este contexto, la presente investigación evalúa la aplicación de la NIA 220-Control de la Calidad de la Auditoría de los Estados Financieros y su relación con la NICC 1, analizando las propuestas para la mejora de la normativa internacional. Además, se expone cuál es la situación del trabajo actual de la auditoría en el mundo, mencionando casos de otros países en donde existen organismos de control establecidos, así como se recopilan opiniones internacionales respecto de la importancia de la calidad de los trabajos de auditoría. Por último, se atiende al análisis de la adopción de la normativa en el Perú, cuál ha sido su evolución en los últimos años y cuáles son los organismos de control de auditoría existentes.

Palabras clave: auditoría, calidad del trabajo de auditoría, NIA 220, NICC 1

\section{Regulation on the quality of audit work: global analysis for a local context (Peru)}

\begin{abstract}
The events that have taken place in recent years with regard to fraud and corruption have caused countries to seek mechanisms that safeguard financial information, firstly through compliance with regulations issued by organizations such as IFAC that promote the application of International Financial Reporting Standards (IFRS). The present in-
\end{abstract}


vestigation analyzes the application of the NIA 220 Control of the Quality of the Audit of the Financial Statements and its relation with the NICC 1, analyzing the proposals for the improvement of the international regulation. We also explain the current status of the audit work in the world, mentioning cases from other countries where there are established control bodies, as well as gathering opinions from other countries regarding the importance of the quality of the audit work. We conclude with the analysis of the adoption of the regulations in Peru, what has been the evolution in recent years and what are the existing audit control bodies.

Keywords: audit, quality of audit work, NIA 220, NICC 1

\section{Regulamento sobre a qualidade do trabalho de au- ditoria: análise global para um contexto local (Peru)}

\section{Resumo}

Nos últimos anos, os eventos sobre fraude e corrupção levaram os países a buscar mecanismos para salvaguardar a informação financeira, em primeiro lugar, através do cumprimento das normas emitidas por organizações como a IFAC, que promove a implementação das Normas Internacionais de Informações Financeiras (IFRS), as Normas Internacionais de Auditoria (NIA) e as Normas Internacionais de Controle de Qualidade (NICC). Neste contexto, a presente investigação avalia a aplicação do ISA 220 - Controle da Qualidade da Auditoria das Demonstrações Financeiras e sua relação com o NICC 1, analisando as propostas para a melhoria da regulação internacional. Além disso, expõe-se a situação do atual trabalho de auditoria no mundo, mencionando casos de outros países onde existem órgãos de controle estabelecidos, bem como reunindo opiniões internacionais sobre a importância da qualidade do trabalho de auditoria. Por fim, aborda-se a análise da adoção de regulamentos no Peru, qual tem sido sua evolução nos últimos anos e quais são os órgãos de controle de auditoria existentes.

Palavras-chave: auditoria, qualidade do trabalho de auditoria, NIA 220, NICC 1 


\section{Introducción}

Los cambios permanentes, producto de la globalización, han generado efectos positivos y negativos en el ámbito empresarial, por lo que es fundamental destacar la calidad del trabajo de auditoría. En ese sentido, los actores principales, como las firmas, los organismos reguladores y, finalmente, pero no menos importante, el Estado, poseen la gran tarea de establecer mecanismos que permitan garantizar la calidad del trabajo de auditoría.

El propósito de este artículo es analizar el marco normativo internacional, así como las últimas propuestas presentadas por los organismos reguladores, a fin de señalar si hoy realmente existe una auditoría para las empresas auditoras.

\subsection{Definición del problema}

Los organismos internacionales, como la IFAC y el AIC, cumplen un papel importante al regularizar la contabilidad, así como la actuación del profesional contable y del auditor con el propósito de garantizar una información de calidad al grupo de interés. El cuestionamiento a las compañías, al profesional contable como constructor de la información de acuerdo a los hechos económicos y al auditor, que da su opinión respecto de la información financiera, es cada vez más notoria. De este modo, los organismos internacionales se han enfocado en definir, redefinir y modificar los lineamientos, así como en regular, cada vez más, la actividad profesional.

Respecto de la actividad del auditor, que es materia de investigación, la IFAC ha emitido una serie de modificaciones a la norma de calidad del trabajo de auditoría, a fin de garantizar la transparencia de la actividad en beneficio de la sociedad. En ese sentido, es fundamental analizar estos cambios en función de los últimos acontecimientos mundiales.

\subsection{Objetivos de la investigación}

Analizar el contexto actual de la regulación de la calidad del trabajo de auditoría a nivel internacional y local

\section{Objetivos Específicos}

- Analizar la normatividad vigente, las últimas modificaciones y discusiones

- Analizar las experiencias de otros países sobre su regulación

\subsection{Justificación e importancia de la investigación}

El presente artículo considera como tema al control de la calidad del trabajo de auditoría, el cual ha resultado relevante en estas últimas décadas, producto de escándalos por fraudes contables, que las auditoras han avalado y que, en consecuencia, han generado millonarias pérdidas a los inversionistas y a la sociedad en general. Bajo esta premisa, el objetivo del presente artículo es exponer un análisis minucio- 
so, por un lado, sobre la regulación y las propuestas presentadas por los organismos internacionales, $y$, por otro, sobre casos en países donde se han tomado acciones y medidas drásticas para la regulación de la actividad de las firmas de auditoría y de profesionales independientes. En este sentido, con la presente investigación, se brinda un aporte presentando un análisis de la regulación internacional, así como el juicio de los autores sobre algunas experiencias internacionales de auditoría.

\section{Metodología}

La metodología aplicada es analítica descriptiva sobre la base de la revisión bibliográfica de distintos autores, de la normativa internacional vigente y de las propuestas planteadas. En esta línea, se describen los conceptos relevantes para contextualizar y analizar las regulaciones internacionales sobre el control de calidad del trabajo de auditoría mediante la identificación de experiencias de varios países que han tomado acciones a fin de mejorar los procedimientos de control en las firmas de auditoría. Asimismo, se determinan las conclusiones del artículo y sus aportes finales.

\section{Marco normativo}

\subsection{Marco normativo internacional}

\subsubsection{El control de calidad basado en las Normas Internacionales del Control de Calidad - NICC1}

De acuerdo al párrafo 11 de la NICC 1, el objetivo es establecer un sistema de control de calidad en las firmas de auditoría, es decir, "proporcionar seguridad razonable del cumplimiento de las normas profesionales, requerimientos legales y reglamentarios aplicables, y, por lo tanto, concluir que los informes emitidos son adecuados en función de las circunstancias". Asimismo, en el párrafo 16, se establecen los siguientes elementos, los cuales deberán ser comunicados a todo el personal:

- Responsabilidades de liderazgo en la calidad dentro de la firma de auditoría

- Requerimientos de ética aplicables

- Aceptación y continuidad de las relaciones con los clientes, y de encargos específicos

- Recursos Humanos

- Realización de encargos

- Seguimiento 


\subsubsection{Control de calidad de la auditoría de estados financieros (NIA 220)}

Esta norma trata sobre las responsabilidades que posee el auditor con respecto a los procedimientos de control de calidad de una auditoría de estados financieros y las responsabilidades del revisor de control de calidad del encargo. Se analiza conjuntamente con las NICC y los requerimientos éticos aplicables.

\subsubsection{Programa Estrategia para 2015 - 2019 y el Plan de Trabajo para 2015 - 2016}

Ante la rápida evolución de la normativa contable y el aumento de las expectativas por parte de los interesados, se torna imprescindible la aplicación de la Norma Internacional de Control de Calidad 1 (NICC 1)- Control de calidad para firmas que realizan auditorías y revisiones de estados financieros, así como otros encargos que proporcionan un grado de seguridad y de servicios relacionados. Estas políticas y procedimientos de control de calidad de la NICC 1 son aplicables a las características y tamaño de cada empresa.

\subsubsection{Plan de Trabajo del IAASB 2015 - 2016}

El IAASB ha propuesto que se deberán atender las cuestiones relacionadas con la NICC 1 basándose en los comentarios surgidos con respecto a los desafíos de implementación que enfrentan algunos profesionales. En respuesta a las preocupaciones de los interesados, el IAASB incluye una nueva iniciativa sobre el control de calidad en su programa Estrategia para 2015 - 2019 y en el Plan de Trabajo para 2015 -2016 "Mejoramiento de la Calidad de la Auditoría y Preparándose para el futuro". Su objetivo estratégico está centrado en garantizar la aplicación de las NIA como base para las auditorías. Por ello, el programa se focaliza en 3 elementos clave: el escepticismo profesional, el control de calidad del trabajo y los grupos de auditoría.

Asimismo, el IAASB ha publicado recientemente el Manual de Control Internacional de Calidad, Auditoría, Revisión, otras Garantías y Servicios, que, en el volumen III, incluye el Marco para Calidad de Auditoría del IAASB. Este especifica los factores clave que crean un ambiente para obtener calidad en la auditoría, mientras que, como visión de conjunto, atiende a los siguientes aspectos:

- La calidad considera que las auditorías se desarrollan de manera consistente.

- El equipo de trabajo de calidad demuestra valores y ética; posee experiencia y realiza el trabajo en el tiempo asignado de acuerdo a la planificación; aplica un riguroso proceso, cumpliendo con las normas establecidas; entrega, en tiempo oportuno, los informes; e interactúa adecuadamente con los interesados.

- La calidad de la auditoría se obtiene en un clima donde exista una cadena correcta de suministro de información financiera. 


\subsubsection{El nuevo enfoque de gestión de calidad}

Este enfoque enfatiza la responsabilidad de los líderes de las firmas de auditoría para brindar una respuesta más proactiva, escalable y sólida para la gestión de riesgos de calidad, la cual sería más fácilmente adaptable al cambiante entorno empresarial. El enfoque de gestión de calidad permitirá integrar las políticas y procedimientos de una entidad dentro de su sistema de calidad, incrementar el interés en la NICC 1 , crear una cultura organizacional en la empresa, establecer los lineamientos para detectar y controlar los riesgos, y diseñar e implementar un efectivo sistema de gestión de la calidad. Asimismo, los elementos que se reconocen son los siguientes: establecer los objetivos de la calidad; efectuar la evaluación de los riesgos, y diseñar e implementar respuestas ante ellos; implementar las actividades de control; e informar, comunicar, documentar y controlar la calidad.

\subsubsection{Futuros cambios en la NICC 1}

Al añadir el enfoque de gestión de calidad en la NICC 1, se generarían cambios centrales, referentes a los siguientes temas:

- Riesgo de incumplir con los objetivos de calidad

- Monitoreo de la calidad de todas las fuentes

- Escalabilidad y auditoría

- No contexto de auditoría

\subsubsection{Enfoque actual y enfoque propuesto}

El enfoque actual constituye un enfoque estandarizado que no siempre se ajusta al sistema de control de calidad de la empresa ni a la adaptación a los cambios del entorno. Incluye los requisitos relacionados con la supervisión, centrándose en los resultados de las inspecciones internas y revisiones por colegas profesionales. Además, los requisitos individuales no contienen material explicativo que ayude a las empresas de todos los tamaños en el diseño de políticas y procedimientos adecuados ni en el desempeño del trabajo de auditoría, en la medida en que presta menos atención a los requisitos de otros compromisos.

Por su parte, el enfoque propuesto describiría acciones para identificar los riesgos considerando la evolución del entorno, y permitiendo diseñar y aplicar procedimientos para enfrentarlos. Abordaría el seguimiento de medidas correctivas aplicadas para subsanar las deficiencias detectadas y promovería, además de las inspecciones, otros tipos de monitoreo, como la pre y post emisión de opiniones. En paralelo, facilitaría el reconocimiento de políticas y procedimientos dependiendo de la naturaleza y la evaluación de los riesgos, apoyando al logro de los objetivos de calidad, y permitiría a la firma considerar objetivos de calidad y riesgos relacionados no solo con la auditoría, sino también con compromisos, como aseguramientos, compilaciones, procedimientos acordados, etc. 


\subsubsection{Opiniones emitidas por los profesionales en relación con el enfo- que propuesto}

En diciembre del 2015, el IAASB publicó el documento de consulta "Invitación a comentar: mejora de la calidad de auditoría en el interés público: un enfoque en escepticismo profesional, control de calidad y auditorías de grupo", con el objetivo de brindar a los profesionales la oportunidad de emitir sus opiniones en torno a la propuesta. Los comentarios de algunos profesionales fueron los siguientes:

\section{- American Institute of CPAs (AICPA)}

Se debe incorporar el enfoque como un principio general que sustente el sistema control de calidad, así como las políticas y procedimientos de la firma. Asimismo, se debe brindar material de apoyo para el diseño de este sistema, el cual permite identificar y responder ante los riesgos.

\section{- Asociación Interamericana de Contabilidad (AIC)}

Mejoraría la calidad de las auditorías, cuyo mayor desafío será la difusión y formación, así como comprender el alcance de las modificaciones.

\section{- Federación Argentina de Consejo Profesionales de Ciencias Económicas (FA- CPCE)}

La dirección de la firma se involucraría en el proceso continuo de evaluación de los riesgos de calidad. Sería la mejor solución para tratar las diferencias en los tamaños de las empresas y los servicios prestados.

\section{- BDO International Limited}

Presenta la capacidad de apoyar la calidad de auditoría sobre una base escalable y sólida. Considera la variedad de las estructuras organizacionales y exige un mayor nivel de compromiso en el trabajo en equipo.

\section{- CPA Australia}

Apoya los esfuerzos para que la calidad en la auditoría pueda mejorar a través del establecimiento de normas y otras actividades que puedan desarrollarse en beneficio de una información transparente.

\section{- Chartered Accountans Ireland}

Consideran la importancia de mejorar la calidad de la auditoría, siendo necesario el establecimiento de medidas para lograr este objetivo. Además, existe una preocupación por la repercusión del costo en los cambios para la obtención de la calidad, lo cual afectaría significativamente a las empresas medianas y pequeñas.

- IFIAR - International Forum of Independent Audit Regulators

La calidad depende de la comunicación del trabajo en grupo y del auditor. Es de mucha importancia para la obtención de un óptimo resultado. 


\section{Situación actual en el mundo del trabajo de auditoría}

Existen denodados esfuerzos tanto de las entidades gubernamentales como de los organismos reguladores en establecer mecanismos de supervisión a fin de asegurar que las grandes sociedades auditoras cumplan las NIA y garanticen su trabajo con calidad. A continuación, se enlistan algunos casos internacionales.

\subsection{Reino Unido}

Financial Reporting Council (FRC) ${ }^{1}$ publicó su informe anual 2015-2016. Mediante su programa de inspección, señala que, en general, la calidad de auditoría en el país es buena, sobre todo en función de los informes de auditoría de las empresas que cotizan en bolsa. No obstante, los resultados muestran que más del $95 \%$ de los auditores, el $91 \%$ de los consejeros y el $73 \%$ de los inversionistas presentan altos niveles de calidad en los informes corporativos, aunque existe una preocupación constante por la longitud y acceso del informe anual y las cuentas.

\subsection{Australia}

La Comisión de Seguridad e Inversiones (ASIC por sus siglas en inglés) ${ }^{2}$ se encarga de inspeccionar a empresas que auditan entidades de interés público, así como a empresas pequeñas que pertenecen al grupo o que se relacionan a auditorías de compañías que cotizan en bolsa. El informe emitido el 2015-2016³, producto de las inspecciones, se focaliza en cuatro áreas que continúan siendo la necesidad de mejora de las firmas de auditoría:

- La suficiencia y propiedad de la evidencia de auditoría obtenida por el auditor

- El nivel profesional de escepticismo ejercido por los auditores

- Uso apropiado en el trabajo de expertos y otros auditores

- Asegurar que las deficiencias materiales detectadas son abordadas o debidamente comunicadas mediante el informe de auditoría

1 . Financial Reporting Council: organismo en el Reino Unido responsable de promover la gestión empresarial de alta calidad y la presentación de informes para fomentar la inversión, aasí como de supervisar y tomar medidas para promover la calidad de la información y la auditoría. Posee facultad para disponer acciones disciplinarias, y supervisar las actividades de contabilidad y firmas auditoras. El Equipo de Auditoría de Revisión de Calidad (AQR) contribuye a este objetivo mediante el control y la promoción de calidad de la auditoría.

2 . Australian Securities and Investments Commission (ASIC) es un ente regulador del gobierno con independencia. Contribuye a la reputación económica de Australia y al bienestar, generando confianza al usuario, y garantizando que los mercados financieros sean justos y transparentes.

3 . Reporte anual 2015-2016 emitido por el ASIC. Archivo disponible en PDF en: https://cdn.tspace.gov. au/uploads/sites/46/2016/10/FRC_Annual_Report_2015-16.pdf 


\subsection{Estados Unidos}

Public Company Accounting Oversight Board (PCAOB) ${ }^{4}$ supervisa las auditorías de las empresas públicas, incluyendo los corredores y distribuidores. Su propósito es proteger al inversionista, mejorar la calidad de la auditoría y la aplicación del correcto ejercicio profesional. En el 2015, se examinaron 75 empresas que cubrían partes de 115 auditorías y contratos de certificación relacionados. Los compromisos de certificación comprendían 27 relacionados al cumplimiento de informes y 87 relativos a los informes de exención.

\subsection{España}

Por medio del Instituto de Contabilidad y Auditoría de Cuentas (ICAC), dentro de su competencia "Control de la actividad" y mediante los sistemas de control de calidad e investigaciones, ejerce la potestad sancionadora aplicable a los auditores de cuentas y a las sociedades de auditoría, entre otras que no son de relevancia para el presente análisis. En el 2015, España publicó su memoria anual, donde, a través de su cuerpo técnico, se encargó de investigar los trabajos de auditoría de las firmas auditoras y auditores independientes. En la memoria, reportaron el número de actuaciones realizadas: 84 inspecciones, 35 investigaciones, y 52 denuncias analizadas y archivadas.

\subsection{Foro Internacional de Reguladores en auditoría independiente (IFIAR)}

IFIAR ${ }^{5}$ es un organismo de alcance internacional que presenta como propósito fundamental servir al interés del público y a la protección del inversor. Brinda una plataforma (foro) a sus miembros para el diálogo e intercambio de información, relacionada con el entorno de la auditoría, que enfatiza la calidad y las prácticas reguladoras de la actividad.

\section{Adopción e implementación normativa en el Perú}

En el Perú, conforme al Decreto Ley 26126 - Ley Orgánica de la Superintendencia de Mercado de Valores, se señala como atribución supervisar el cumplimiento de las Normas Internacionales de Auditoría por parte de las sociedades auditoras habilitadas por un colegio profesional. Asimismo, mediante la Ley 29720-2011 del 25/06/2011- Ley que promueve las emisiones de valores mobiliarios y fortalece el

4 . PCAOB es un organismo sin fines de lucro establecido por la Ley Sarbanes-Oxley de 2002. Esta requiere que el PCAOB presente un informe anual, que incluye a los estados financieros auditados, a la Comisión de Bolsa y Valores (SEC) para su transmisión al Comité del Senado sobre Banca, Vivienda y Asuntos Urbanos, y al Comité de Servicios Financieros. Consultado en la web: https://pcaobus.org/Pages/default. aspx

5 . IFIAR (International Forum of Independent Audit Regulators) es un foro internacional creado el 2006. Posee representación en 51 jurisdicciones, África, América del Norte, América del Sur, Asia, Oceanía y Europa. Consultado en sitio web: www.ifiar.org 
mercado de capitales, en el artículo 5, se señalaba que las empresas no supervisadas por la SMV, que excedan en ingresos o activos totales las 3000 UIT, deberían presentar estados financieros auditados. Luego, mediante resoluciones de superintendencia, se incorporaron los plazos para la presentación. Así, en la resolución 0002-2016 del 30/01/2016, se establecieron nuevos plazos y tramos para la presentación de los estados auditados y de adopción por primera vez de las NIIF.

Conforme al Expediente Nro. 0009-2014-PI/TC, en abril del 2016, el Tribunal Constitucional declara fundada la demanda de inconstitucionalidad respecto de la obligación de presentar los estados financieros auditados para entidades que no se encuentran bajo la supervisión de la SMV. Este aspecto normativo dificulta, en la actualidad, la implementación de las NIIF y de las NIA en la mayoría de empresas en el Perú, puesto que la norma, en el artículo 5 de la Ley 29720, indica también los plazos para la adopción de las NIIF, lo que dificulta el compromiso de la Junta de Decanos de los Colegios Profesionales de Contabilidad con las entidades internacionales.

\subsection{Organismos de supervisión en el país}

En el Perú, el ente encargado de recibir la información financiera de las empresas que cotizan en bolsa es la SMV. Esta información es recibida y evaluada permanente salvaguardando los intereses de los accionistas e inversores. Luego de analizarla, debe publicarla para que se encuentre a disposición de los interesados. A nivel nacional, finalizando el 2016, existían 2 029,648 empresas, según el INEI. En otras palabras, casi la totalidad de empresas no era supervisada financieramente; solamente en el caso de acceso al sistema financiero, es evaluada al momento de adquirir algún producto bancario. De esto, se desprende que no existe un organismo que vele por la supervisión de la calidad de las auditorías en el Perú, conforme se establece en la NIA 220.

\section{Conclusiones y/o aportes}

- La incorporación del enfoque de gestión de calidad a la NICC 1 busca mejorar la calidad del trabajo de auditoría a nivel empresarial en las firmas de auditoría, en vinculación con los objetivos estratégicos de la entidad y bajo la responsabilidad directa de un liderazgo firme, que destaque los aspectos claves que conducen a mejor la calidad del trabajo.

- El socio del trabajo es responsable de la calidad general del trabajo de auditoría y la firma de auditoría se ha asegurado de sus capacidades para la realización del trabajo de auditoría asignado. Por lo tanto, es importante proporcionar orientación a las firmas de auditoría sobre cómo efectuar un mejor trabajo sobre la base de los principios de gobierno corporativo con el fin de promover la calidad de la auditoría.

- Gran parte de los países desarrollados cuentan con un rol activo en torno al trabajo de auditoría y vienen sumando esfuerzos mediante el establecimiento de 
alianzas, compartiendo información y experiencias a través del IFIAR.

- Los organismos como PCAOB, FRC, ASIC, ICAC, entre otros, poseen facultades respaldadas por ley para inspeccionar, vigilar y definir acciones que promuevan la mejora de la calidad del trabajo de auditoría en pos de proteger al inversor, lo cual implica que el Estado cumple un papel fundamental.

- Existe una necesidad latente del público interesado que exige transparencia en la información financiera y el auditor posee una responsabilidad importante frente la sociedad.

- Los informes publicados de los diferentes organismos de inspección muestran hallazgos recurrentes y comunes: problemas de estimación, reconocimiento de ingresos, falta de consistencia en sus políticas y procedimientos de control.

- La solidez, consistencia, coherencia y la mejora continua de los sistemas de control de calidad son fundamentales en las firmas de auditoría para obtener un trabajo de auditoría razonable y de calidad.

- La actividad de la auditoría es necesaria y útil para la sociedad, en tanto que el profesional o las firmas venden confianza.

- A juicio de los autores, se debe considerar las experiencias analizadas en este trabajo a fin de tomar iniciativas desde el Estado hasta los colegios profesionales del país, para la implementación de una entidad, como existe en otros países, que vele por la calidad del trabajo de auditoría en el Perú.

\section{REFERENCIAS BIBLIOGRÁFICAS}

IAASB. (2016). Focus on Audit Quality. Recuperado de: http://www.iaasb.org/focus-audit-quality.

IAASB. (2015). Invitación a Comentar: Mejora de la calidad de auditoría en el Interés Público: Un Enfoque en Escepticismo profesional, control de calidad y auditorías de grupo. Recuperado de: http://www.ifac.org/publications-resources/invitation-comment-enhancing-audit-quality-public-interest

Arnold, C. (2016). An SMP Perspective on Improving the Structure of the IESBA Code of Ethics for Professional Accountants_Phase 1. Recuperado de: http://www. ifac.org/global-knowledge-gateway/ethics/discussion/smp-perspective-improving-structure-iesba-code-ethics-0

IFAC. (2009). Normas Internacionales de Control de Calidad 1 (NICC1). Recuperado de: https://www.ifac.org

IFAC. (2015). Guía de control de calidad para pequeñas y medianas firmas de auditoría, 3ra edición. Recuperado de: https://www.ifac.org 
IFAC. (2016). Manual de Control Internacional de Calidad, Auditoría, Revisión, otras Garantías y Servicios, edición 2016-2017. Recuperado de: https://www.ifac.org

Instituto de Contabilidad y Auditoría de Cuentas. (2014). Memoria anual de actividades 2015. Recuperado de: http://www.icac.meh.es/Documentos/ebook/Memoria/2015/es/index.html

PCAOB (Public Company Accounting Oversight Board). (2015). Annual report 2015. Pdf disponible en: https:

//pcaobus.org/About/Administration/Documents/Annual\%20Reports/2015.pdf

ASIC (Australian Securities and Investments Commission). (2015). Annual report 2014-2015. Pdf disponible en: https://cdn.tspace.gov.au/uploads/sites/46/2016/10/ FRC_Annual_Report_2015-16.pdf

IFIAR. (2015). Annual Report 2015. Pdf disponible en: https://www.ifiar.org/IFIAR/ media/Documents/General/About\%20Us/IFIAR-2015-Annual-Report.pdf

Fecha de recepción: $31 / 10 / 2018$

Fecha de aceptación: 21/11/2018

Correspondencia: cpcalvarezlopez@gmail.com

Imorales.ngc@gmail.con 